\title{
Action principle for so-called non-Lagrangian systems
}

\section{D.M. Gitman*†}

Instituto de Física, Universidade de São Paulo, Brazil

E-mail: gitman@dfn.if.usp.br

\section{V.G. Kupriyanov}

Instituto de Física, Universidade de São Paulo, Brazil

Physics Department, Tomsk State University, Russia

E-mail: kvgedfn.if.usp.br

We consider the problem of constructing an action functional for physical systems whose classical equations of motion cannot be directly identified with Euler-Lagrange equations for an action principle. The existence of an action principle for a given physical system, or what is the same, the existence of a Lagrange function for such a system, allows one to proceed with canonical quantization schemes. This, in particular, emphasizes the importance of formulating an action principle for any physical system. From simple consideration, we find necessary and sufficient conditions for the existence of a multiplier matrix which can endow a prescribed set of second-order equations with the structure of Euler-Lagrange equations. An explicit form of the Lagrangian is constructed for a system which admits the existence of such a multiplier. If a given set of differential equations cannot be derived from an action principle, one can reformulate such a set in an equivalent first-order form which can always be treated as the Euler-Lagrange equations of a certain action. We construct such an action explicitly. There exists an ambiguity (not reduced to a total time derivative) in associating a Lagrange function with a given set of equations. We present a complete description of this ambiguity. The general procedure is illustrated by several examples.

Fifth International Conference on Mathematical Methods in Physics - IC2006

April 24-28 2006

Centro Brasileiro de Pesquisas Fisicas, Rio de Janeiro, Brazil

\footnotetext{
* Speaker.

Gitman is grateful to the Brazilian foundations FAPESP and CNPq for permanent support.

${ }^{\ddagger}$ Kupriyanov thanks FAPESP for support
} 


\section{Introduction}

The problem of constructing an action functional for a given set of differential equations is known in literature as the inverse problem of the calculus of variations for Newtonian mechanics. This problem has been under consideration for more then hundred years. As early as 1887 Helmholtz [1] presented a criterion of commutativity for second variational derivatives with the help of which one can define whether a set of differential equations Lagrangian or not. In 1894 Darboux [2] solved the problem for one dimensional case. In 1941 the case of two degrees of freedom was investigated by Douglas [3]; in particular, he presented examples of second-order equations which cannot be obtained from the variational principle. Afterwards there were numerous works devoted to the development a general theory for multidimensional systems (see e.g., [4]-[18] and references therein).

In the present work we attempt to settle some general points of such a theory. We construct an action principle for a given system of second-order differential equations using so-called integrating multiplier [3]-[6], i.e., such a nonsingular matrix which being multiplied by a given set of equations reduces it to a standard Euler-Lagrange form. In section 2 we present a simple derivation method for necessary and sufficient conditions for a integrating multiplier, which does not appeal to the theory of generalized functions. We also construct the explicit form of Lagrangian in a case an integrating multiplier does exist and is known. Then we apply our method for investigating the inverse problem of the calculus of variations for some simple models. We construct an action principle for multidimensional dissipative systems. We also consider an example of a linear dynamical system whose equations of motion do not admit the existence of an integrating multiplier and as a consequence cannot be obtained from the minimum action principle.

Note that it is always possible to reduce non-Lagrangian second-order equations of motion to an equivalent set of first-order differential equations. For such equations, one can always construct an action principle, the corresponding consideration is represented in section 3 and, partially, is based on results of works [14] and [18]. Thus, we show that systems traditionally called nonLagrangian ones are, in fact, equivalent to some first-order Lagrangian systems. As an example, we construct a first-order action functional for any linear dynamical system.

\section{Action functional for a set of second-order equations}

\subsection{General consideration}

Let a system with $n$ degrees of freedom be described by a set of $n$ second-order differential equations of motion, solvable with respect to second-order time derivatives. Suppose such a set has a form

$$
\ddot{q}^{i}-f^{i}(t, q, \dot{q})=0, \quad i=1 \ldots n
$$

where $f^{i}(t, q, \dot{q})$ are some functions of the indicated arguments, and by dots above we denote time derivatives of the coordinates. Let us construct an action principle for this set. If (2.1) cannot be directly identified with Euler-Lagrange equations, one can find an integrating multiplier, i.e., such a nonsingular matrix $h_{i j}(t, q, \dot{q})$ that being multiplied by $(2.1)$

$$
h_{i j}\left[\ddot{q}^{j}-f^{j}(t, q, \dot{q})\right]=0
$$


reduces this set to a standard Euler-Lagrange form for some Lagrangian $L(t, q, \dot{q})$,

$$
\frac{\partial L}{\partial q^{i}}-\frac{\partial^{2} L}{\partial t \partial \dot{q}^{i}}-\frac{\partial^{2} L}{\partial \dot{q}^{i} \partial q^{j}} \dot{q}^{j}-\frac{\partial^{2} L}{\partial \dot{q}^{i} \partial \dot{q}^{j}} \ddot{q}^{j}=0 .
$$

In order to identify (2.2) with (2.3) we need to ensure that

$$
\begin{aligned}
& \frac{\partial^{2} L}{\partial \dot{q}^{i} \partial \dot{q}^{j}}=h_{i j}, \\
& \frac{\partial L}{\partial q^{i}}-\frac{\partial^{2} L}{\partial t \partial \dot{q}^{i}}-\frac{\partial^{2} L}{\partial \dot{q}^{i} \partial q^{j}} \dot{q}^{j}=h_{i j} f^{j}
\end{aligned}
$$

Provided that an integrating multiplier is known, equations (2.4)-(2.5) can be interpreted as a system of equations for a Lagrange function $L$. We are going to solve the set of equations (2.4)-(2.5). Its consistency conditions will give us all the necessary and sufficient conditions for an integrating multiplier. Assuming that $L$ is a smooth function of the indicated arguments, the consistency condition for equation (2.4) imply that

$$
h_{i j}=h_{j i}, \quad \frac{\partial h_{i j}}{\partial \dot{q}^{k}}=\frac{\partial h_{k j}}{\partial \dot{q}^{i}} .
$$

If (2.6) does hold one can solve equation (2.4). To this end, we remind that the general solution of the equation $\partial f / \partial q^{i}=g_{i}$, provided the vector $g_{i}$ is a gradient, is

$$
f(q)=\int_{0}^{1} d s q^{i} g_{i}(s q)+c
$$

where $c$ is a constant. Taking the above fact into account, we obtain for $L$ (we do not consider global problems which can arise from non-trivial topology of the configuration space) the following representation:

$$
L=K(t, q, \dot{q})+l_{i}(t, q) \dot{q}^{i}+l_{0}(t, q)
$$

where

$$
K(t, q, \dot{q})=\int_{0}^{1} d a \dot{q}^{j}\left[\int_{0}^{1} d b \dot{q}_{1}^{i} h_{i j}\left(t, q, b \dot{q}_{1}\right)\right]_{\dot{q}_{1}=a \dot{q}}
$$

and $l_{0}(t, q), l_{i}(t, q)$ are some functions of the indicated arguments. To find these functions, we use equation (2.5). Substituting (2.7) into (2.5), we get

$$
\frac{\partial K}{\partial q^{i}}-\frac{\partial^{2} K}{\partial \dot{q}^{i} \partial t}-\frac{\partial^{2} K}{\partial \dot{q}^{i} \partial q^{j}} \dot{q}^{j}+\left(\frac{\partial l_{j}}{\partial q^{i}}-\frac{\partial l_{i}}{\partial q^{j}}\right) \dot{q}^{j}-\frac{\partial l_{i}}{\partial t}+\frac{\partial l_{0}}{\partial q^{i}}=h_{i j} f^{j} .
$$

Differentiating this equation over $\dot{q}^{k}$, we obtain:

$$
\frac{\partial l_{k}}{\partial q^{i}}-\frac{\partial l_{i}}{\partial q^{k}}=L_{i k}
$$

where

$$
L_{i k}=\frac{\partial^{2} K}{\partial \dot{q}^{i} \partial q^{j}}-\frac{\partial^{2} K}{\partial \dot{q}^{i} \partial q^{j}}+\frac{\partial h_{i k}}{\partial t}+\dot{q}^{j} \frac{\partial h_{i k}}{\partial q^{j}}+\frac{\partial}{\partial \dot{q}^{k}}\left(h_{i j} f^{j}\right)
$$


This equation is a differential equation for $l_{i}$. The consistency conditions of equation (2.10) imply, that first of all, the symmetric part of $L_{i k}$ is zero, which can be written as

$$
\hat{D} h_{i k}+\frac{1}{2}\left(h_{i j} \frac{\partial f^{j}}{\partial \dot{q}^{k}}+h_{k j} \frac{\partial f^{j}}{\partial \dot{q}^{i}}\right)=0,
$$

where

$$
\hat{D}=\frac{\partial}{\partial t}+\dot{q}^{j} \frac{\partial}{\partial q^{j}}+f^{j} \frac{\partial}{\partial \dot{q}^{j}}
$$

Using (2.12), one can rewrite (2.11) as follows

$$
L_{i k}=\frac{\partial^{2} K}{\partial \dot{q}^{i} \partial q^{k}}-\frac{\partial^{2} K}{\partial \dot{q}^{k} \partial q^{i}}+A_{i k}, A_{i k}=\frac{1}{2}\left(h_{i j} \frac{\partial f^{j}}{\partial \dot{q}^{k}}-h_{k j} \frac{\partial f^{j}}{\partial \dot{q}^{i}}\right)
$$

Next, $L_{i k}$ does not depend on velocities, i.e., $\partial L_{i k} / \partial \dot{q}^{l}=0$, which yields

$$
\frac{\partial h_{k l}}{\partial q^{i}}-\frac{\partial h_{i l}}{\partial q^{k}}=\frac{\partial}{\partial \dot{q}^{l}} A_{i k}
$$

And, finally, the Jacobi identity

$$
\frac{\partial L_{i k}}{\partial q^{l}}+\frac{\partial L_{k l}}{\partial q^{i}}+\frac{\partial L_{l i}}{\partial q^{k}}=0 \Rightarrow \frac{\partial A_{i k}}{\partial q^{l}}+\frac{\partial A_{k l}}{\partial q^{i}}+\frac{\partial A_{l i}}{\partial q^{k}}=0
$$

Provided $h_{i j}$ obeys equations (2.12), (2.14) and (2.15), $l_{i}$ can be found from equation (2.10). We remind that the general solution for $l_{i}$ of equation (2.10) is given by

$$
l_{i}(t, q)=\int_{0}^{1} d a q^{k} L_{k i}(t, a q)+\frac{\partial \varphi(t, q)}{\partial q^{i}}
$$

where $\varphi(t, q)$ is an arbitrary function.

Now from equation (2.9) we can find $l_{0}$; to this end let us rewrite it as follows:

$$
\frac{\partial l_{0}}{\partial q^{i}}=m_{i}
$$

where

$$
m_{i}=h_{i j} f^{j}-\frac{\partial K}{\partial q^{i}}+\frac{\partial^{2} K}{\partial t \partial \dot{q}^{i}}+\dot{q}^{j} \frac{\partial^{2} K}{\partial q^{j} \partial \dot{q}^{i}}-\dot{q}^{j} L_{i j}+\frac{\partial l_{i}}{\partial t} .
$$

The consistency conditions of (2.17) imply, that, first, $m_{i}$ does not depend on velocities, i.e., $\partial m_{i} / \partial \dot{q}^{k}=0$. This condition is provided by equation (2.12). And second, the vector $m_{i}$ must be a gradient:

$$
\frac{\partial m_{i}}{\partial q^{k}}-\frac{\partial m_{k}}{\partial q^{i}}=\frac{\partial A_{i k}}{\partial t}+\dot{q}^{j} \frac{\partial A_{i k}}{\partial q^{j}}+\frac{\partial}{\partial q^{k}}\left(h_{i j} f^{j}\right)-\frac{\partial}{\partial q^{i}}\left(h_{k j} f^{j}\right)=0 .
$$

Taking into account (2.6), (2.12) and (2.14), one gets from (2.19) the following algebraic condition:

$$
h_{i j} B_{k}^{j}-h_{k j} B_{i}^{j}=0
$$


where

$$
B_{j}^{i}=\frac{1}{2} \frac{\partial f^{i}}{\partial \dot{q}^{m}} \frac{\partial f^{m}}{\partial \dot{q}^{j}}-\hat{D} \frac{\partial f^{i}}{\partial \dot{q}^{j}}+2 \frac{\partial f^{i}}{\partial q^{j}} .
$$

If $h_{i j}$ obeys (2.20), then from (2.17) one gets

$$
l_{0}(t, q)=\int_{0}^{1} d a q^{k} m_{k}(t, a q)+\frac{\partial \varphi(t, q)}{\partial t}+c(t)
$$

where $c(t)$ is an arbitrary function of time.

Thus, we have proved the following statement: iff for a given set of second-order ordinary differential equations (2.1) there exist such a non-singular matrix $h_{i j}(t, q, \dot{q})$ that obeys equations (2.6), (2.12), (2.14), (2.15) and (2.20), then this set can be obtained from the variational principle with the Lagrangian (2.7), where functions $K(t, q, \dot{q}), l_{i}(t, q)$ and $l_{0}(t, q)$ are defined by (2.8), (2.16) and (2.21) correspondingly and the functions $\varphi(t, q)$ and $c(t)$ are arbitrary functions of the indicated arguments.

The arbitrariness related to the functions $\varphi(t, q)$ and $c(t)$ enter Lagrangian (2.7) via the total time derivative of a function $F$,

$$
F=\varphi(t, q)+\int c(t) d t
$$

Note that an integrating multiplier $h_{i j}$, and as a consequence the Lagrange function $L$ does exist, but however not for any set of equations (2.1). In Section 3 we consider an example of a dynamical system which does not admit the existence of an integrating multiplier. However, if it does exist, it is not unique, e.g., if the matrix $h_{i j}$ is an integrating multiplier for a certain set (2.1), it is easy to see that the matrix $h_{i j}=c h_{i j}$, where $c \neq 0$ is a constant, is an integrating multiplier as well. Therefore, Lagrangian (2.7) leading to the set of equations (2.1) is not unique; for this set there exist as many inequivalent Lagrangians as integrating multipliers. Lagrangians corresponding to different integrating multipliers are known as $s$-equivalent Lagrangians.

In the one dimensional case $\ddot{q}-f(t, q, \dot{q})=0$, an integrating multiplier is a non-vanishing function $h(t, q, \dot{q})$ that obeys the equation

$$
\frac{\partial h}{\partial t}+\dot{q} \frac{\partial h}{\partial q}+\frac{\partial}{\partial \dot{q}}(f h)=0 .
$$

This is a first-order partial differential equation which obviously has a solution for any $f$ and initial condition $h(t=0, q, \dot{q})=h_{0}(q, \dot{q})$. As we can see, an answer to the question whether there exist a solution of the inverse problem of the calculus of variations depends on the number of degrees of freedom $n$. For $n=1$ the answer is always positive, and there exist as many inequivalent Lagrangians as functions $h_{0}(q, \dot{q})$ of two variables. For $n \geq 2$ the answer is generally negative.

\subsection{Examples}

In this section we consider a possibility of constructing an action principle for some examples of dynamical systems. First of all, let us consider dissipative systems. Suppose we have an ideal system with the Lagrangian

$$
L_{0}=\frac{\dot{q}^{2}}{2}+V(q), \quad q=\left\{q^{i}\right\}, \quad i=1 \ldots n .
$$


Let us consider the case when besides the potential conservative force $F^{i}=\frac{\partial V}{\partial q^{i}}$ there exist a friction force

$$
F_{\text {fric }}^{i}=\alpha \dot{q}^{i},
$$

where $\alpha$ is a phenomenological friction coefficient which in generally can depend on time. The equations of motion for such a system have the form

$$
\ddot{q}^{i}=\frac{\partial V}{\partial q^{i}}+\alpha \dot{q}^{i}
$$

These equations are non-Lagrangian, but for this set it is possible to find an integrating multiplier. In the simplest case, when it does not depend on coordinates and velocities, it has the form

$$
h_{i j}=e^{-2 \int \alpha d t} h_{i j}^{0}
$$

where $h_{i j}^{0}$ is an arbitrary, symmetric, nonsingular, constant matrix commuting with the matrix $V_{i j}=$ $\partial^{2} V / \partial q^{i} \partial q^{j}$. Using the statement of the previous section, we obtain the following Lagrangian:

$$
L=\frac{1}{2} \dot{q}^{i} h_{i j} \dot{q}^{j}+\int_{0}^{1} q^{i} h_{i j} \frac{\partial V(s \vec{q})}{\partial q^{j}} d s .
$$

If one sets $h_{i j}^{0}=\delta_{i j}$, Lagrangian (2.27) can be rewritten as

$$
L=e^{-2 \int \alpha d t} L_{0}
$$

Note that once the friction coefficient goes to zero, Lagrangian (2.28) transforms into the initial Lagrangian (2.23).

Let us now consider the case when the potential in the initial Lagrangian is linear in velocities. For simplicity we consider the two-dimensional case

$$
L_{0}=\frac{1}{2}\left(\dot{x}^{2}+\dot{y}^{2}+\beta(\dot{x} y-\dot{y} x)\right)
$$

Let us consider this system in the presence of dissipative the force (2.27). The equations of motion will have the form:

$$
\begin{aligned}
& \ddot{x}=\alpha \dot{x}-\beta \dot{y}, \\
& \ddot{y}=\beta \dot{x}+\alpha \dot{y} .
\end{aligned}
$$

As was shown in [19], this system describes a moving charged particle in a uniform magnetic field with radiation friction. In this case,

$$
B_{j}^{i}=\left(\begin{array}{cc}
\alpha^{2}-\beta^{2} & -2 \alpha \beta \\
2 \alpha \beta & \alpha^{2}-\beta^{2}
\end{array}\right)
$$

and from equation (2.20) one immediately gets

$$
\operatorname{tr}\left(h_{i j}\right)=h_{11}+h_{22}=0 .
$$


It is then easy to find that the general solution of the equations (2.6), (2.12), (2.14), (2.15) is defined by an arbitrary function $\phi(\zeta, \eta)$ and has a form

$$
h_{i j}=\left(\begin{array}{cc}
F+\bar{F} & i(F-\bar{F}) \\
i(F-\bar{F}) & -(F+\bar{F})
\end{array}\right),
$$

where $F=\phi\left(\dot{\xi} e^{-\gamma t}, \dot{\xi}-\alpha \xi\right) e^{-\gamma t}, \xi=x+i y, \gamma=\alpha+i \beta$ and the bar denotes a complex conjugation.

The simplest real solution can be found if we put $\phi=1 / \zeta$. We have

$$
h_{i j}=\frac{2}{\dot{x}^{2}+\dot{y}^{2}}\left(\begin{array}{cc}
\dot{x} & \dot{y} \\
\dot{y} & -\dot{x}
\end{array}\right) \text {. }
$$

Using the formulas (2.7), (2.8), (2.16) and (2.21) we find the following Lagrangian:

$$
L=\frac{1}{2} \dot{x} \ln \left(\dot{x}^{2}+\dot{y}^{2}\right)+\dot{y} \arctan \left(\frac{\dot{x}}{\dot{y}}\right)+\alpha x-\beta y .
$$

The corresponding Euler-Lagrange equations

$$
\frac{\ddot{x} \dot{x}+\ddot{y} \dot{y}}{\dot{x}^{2}+\dot{y}^{2}}=\alpha, \frac{\ddot{x} \dot{x}-\ddot{y} \dot{y}}{\dot{x}^{2}+\dot{y}^{2}}=\beta
$$

are equivalent to the initial ones (2.30), with the exception of the point $\dot{x}=\dot{y}=0$. Thus, we can see that in this case the inverse problem of the calculus of variations is solvable. Unfortunately, neither Lagrangian (2.34) nor any other Lagrangian constructed by the matrix (2.32) in the limit $\alpha \rightarrow 0$ transforms into the initial Lagrangian (2.29), modulo a total time derivative. This is because, according to the algebraic condition (2.31), the trace of the Hessian matrix of any Lagrangian for the set of equations (2.30) must be equal to zero and this property holds true after the limit $\alpha \rightarrow 0$ is taken. On the other hand, the trace of the Hessian matrix of the Lagrangian $L_{0}$ in (2.29) is equal to 2. This contradiction proves the statement.

Finally we consider example of dynamical system for which an integrating multiplier and, consequently, the possibility of the Lagrangian description does not exist. Duglas [3] showed that the set of second-order equations

$$
\begin{aligned}
& \ddot{x}+\dot{y}=0, \\
& \ddot{y}+y=0
\end{aligned}
$$

does not admit an integrating multiplier. Let us make sure of this. To this end, let us assume the opposite, namely, let there exist such a non-degenerate matrix $h_{i j}$ that obeys equations (2.6), (2.12), (2.14), (2.15) and (2.20). Then from the algebraic equation (2.20) it follows that $h_{i j}$ must be diagonal $\left(h_{12}=h_{21}=0\right)$, since in this case

$$
B_{j}^{i}=\left(\begin{array}{ll}
0 & 0 \\
0 & 2
\end{array}\right) .
$$

Then, from condition (2.12) we immediately obtains that $h_{11}=0$, and arrive at a contradiction, $\operatorname{det} h_{i j}=0$.

Thus, we can see that an action functional in second-order formalism cannot be constructed not for some sets of differential equations. Nevertheless, as we show in the following section, it is always possible to construct an action principle for the equivalent set of first-order equations. 


\section{Action principle in the first-order form}

Let a system with $n$ degrees of freedom be described by a set of $n$ non-Lagrangian secondorder differential equations of motion. To construct an action principle, we replace these equations (which is always possible) by an equivalent set of $2 n$ first-order differential equations, solvable with respect to time derivatives. Suppose such a set has the form

$$
\dot{x}^{\alpha}=f^{\alpha}(t, x), \alpha=1, . ., 2 n,
$$

where $f^{\alpha}(t, x)$ are some functions of the indicated arguments and by dots stand for time derivatives of coordinates. Since these equations are first-order, action $S[x]$ that yields (3.1) as Euler-Lagrange equations must be linear in the first time derivative $\dot{x}^{\alpha}$. Its general form is

$$
S[x]=\int d t L, L=J_{\alpha} \dot{x}^{\alpha}-H,
$$

where $J_{\alpha}=J_{\alpha}(t, x)$ and $H=H(t, x)$ are some functions of the indicated arguments. The EulerLagrange equations corresponding to (3.2) are

$$
\frac{\delta S}{\delta x}=\frac{\partial L}{\partial x}-\frac{d}{d t} \frac{\partial L}{\partial \dot{x}}=0 \Longrightarrow-\partial_{\alpha} H-\partial_{t} J_{\alpha}+\left(\partial_{\alpha} J_{\beta}-\partial_{\beta} J_{\alpha}\right) \dot{x}^{\beta}=0,
$$

with the notation

$$
\partial_{\alpha}=\frac{\partial}{\partial x^{\alpha}}, \partial_{t}=\frac{\partial}{\partial t} .
$$

Denoting the combination $\left(\partial_{\alpha} J_{\beta}-\partial_{\beta} J_{\alpha}\right)$ by $\Omega_{\alpha \beta}$,

$$
\Omega_{\alpha \beta}=\partial_{\alpha} J_{\beta}-\partial_{\beta} J_{\alpha}=\Omega_{\alpha \beta}(t, x)=-\Omega_{\beta \alpha}(t, x),
$$

we rewrite (3.3) as follows:

$$
\Omega_{\alpha \beta} \dot{x}^{\beta}=\partial_{\alpha} H+\partial_{t} J_{\alpha}
$$

Equations (3.3) or (3.5) can be identified with (3.1), provided that

$$
\begin{aligned}
& \operatorname{det} \Omega_{\alpha \beta} \neq 0, \\
& \Omega_{\alpha \beta} f^{\beta}-\partial_{t} J_{\alpha}=\partial_{\alpha} H .
\end{aligned}
$$

The functions $J_{\alpha}$ and $H$ can be found from conditions (3.4)-(3.7) if the matrix $\Omega_{\alpha \beta}$ is given. Assuming that $J_{\alpha}$ and $H$ are smooth functions the consistency condition for equations (3.7) imply

$$
\partial_{\beta}\left(\Omega_{\alpha \gamma} f^{\gamma}\right)-\partial_{\alpha}\left(\Omega_{\beta \gamma} f^{\gamma}\right)+\partial_{t} \Omega_{\alpha \beta}=0 \Longrightarrow \partial_{t} \Omega_{\alpha \beta}+£_{f} \Omega_{\alpha \beta}=0
$$

where $£_{f} \Omega_{\alpha \beta}$ is the Lie derivative of $\Omega_{\alpha \beta}$ along the vector field $f^{\gamma}$. In addition, one can verify that the matrix $\Omega_{\alpha \beta}$ (3.4) obeys the Jacobi identity $\left(\Omega_{\alpha \beta}\right.$ is a symplectic matrix)

$$
\partial_{\alpha} \Omega_{\beta \gamma}+\partial_{\beta} \Omega_{\gamma \alpha}+\partial_{\gamma} \Omega_{\alpha \beta}=0
$$

Now we are going to analyze these equations. It is known that the general solution $\Omega_{\alpha \beta}$ of equation (3.8) can be constructed with the help of a solution of the Cauchy problem for equations (3.1). Suppose that such a solution is known,

$$
x^{\alpha}=\varphi^{\alpha}\left(t, x_{(0)}\right), x_{(0)}^{\alpha}=\varphi^{\alpha}\left(0, x_{(0)}\right)
$$


being a solution of equations (3.1) for any $x_{(0)}=\left(x_{(0)}^{\alpha}\right)$, and $\chi^{\alpha}(t, x)$ be the inverse function with respect to $\varphi^{\alpha}\left(t, x_{(0)}\right)$, i.e.,

$$
x^{\alpha}=\varphi^{\alpha}\left(t, x_{(0)}\right) \Longrightarrow x_{(0)}^{\alpha}=\chi^{\alpha}(t, x), x^{\alpha} \equiv \varphi^{\alpha}\left(t, \chi^{\alpha}\right),\left.\partial_{\alpha} \chi^{\gamma}\right|_{t=0}=\delta_{\gamma}^{\alpha} .
$$

Then

$$
\Omega_{\alpha \beta}(t, x)=\partial_{\alpha} \chi^{\gamma} \Omega_{\gamma \delta}^{(0)}(\chi) \partial_{\beta} \chi^{\delta}
$$

where the matrix $\Omega_{\alpha \beta}^{(0)}$ is the initial condition for $\Omega_{\alpha \beta}$,

$$
\left.\Omega_{\alpha \beta}(t, x)\right|_{t=0}=\Omega_{\alpha \beta}^{(0)}(x)
$$

It follows from (3.9) at $t=0$ that the matrix $\Omega_{\alpha \beta}^{(0)}(x)$ obeys the Jacobi identity such that the general structure of this matrix is (we do not consider global problems which arise from a nontrivial topology of the $x^{\alpha}$-space)

$$
\Omega_{\alpha \beta}^{(0)}=\partial_{\alpha} j_{\beta}-\partial_{\beta} j_{\alpha}
$$

where $j_{\alpha}(x)$ are some arbitrary functions. Then equation (3.12) implies

$$
\Omega_{\alpha \beta}=\partial_{\alpha} \psi_{\beta}-\partial_{\beta} \psi_{\alpha}, \psi_{\alpha}(t, x)=j_{\beta}(\chi(t, x)) \partial_{\alpha} \chi^{\beta}(t, x) .
$$

On the other hand, relation (3.4) must hold,

$$
\partial_{\alpha} \psi_{\beta}-\partial_{\beta} \psi_{\alpha}=\partial_{\alpha} J_{\beta}-\partial_{\beta} J_{\alpha}
$$

which implies that

$$
J_{\alpha}(t, x)=\psi_{\alpha}+\partial_{\alpha} \varphi=j_{\beta}(\chi(t, x)) \partial_{\alpha} \chi^{\beta}(t, x)+\partial_{\alpha} \varphi(t, x),
$$

where $\varphi(t, x)$ is an arbitrary function. One can represent another form for $J_{\alpha}(t, x)$, in which the ambiguity related to the arbitrary functions $j_{\beta}(x)$ is incorporated in the matrix $\Omega_{\alpha \beta}^{(0)}$. The general solution for $J_{\alpha}(t, x)$ of the equation (3.4) provided that $\Omega_{\alpha \beta}$ is a given antisymmetric matrix that obeys the Jacobi identity, is given by

$$
J_{\alpha}(t, x)=\int_{0}^{1} x^{\beta} \Omega_{\beta \alpha}(t, s x) s d s+\partial_{\alpha} \varphi(t, x),
$$

where $\varphi(x)$ is an arbitrary function. Substituting (3.12) into (3.16), we obtain

$$
J_{\alpha}(t, y)=\left.\int_{0}^{1} y^{\beta}\left[\partial_{\alpha} \chi^{\gamma} \Omega_{\gamma \delta}^{(0)}(\chi) \partial_{\beta} \chi^{\delta}\right]\right|_{x=s y} s d s+\partial_{\alpha} \varphi(t, y)
$$

Equations (3.15) or (??) describe all the ambiguity (arbitrary functions $j_{\beta}(x)$ and $\varphi(t, x)$, or arbitrary symplectic matrix $\Omega_{\gamma \delta}^{(0)}$ and arbitrary function $\varphi(t, x)$ ) in constructing the term $J_{\alpha}(t, x)$ of the Lagrange function (3.2).

One can also see that choosing the matrix $\Omega_{\alpha \beta}^{(0)}(x)$ to be nonsingular, we guarantee the nonsingularity (condition (3.6)) for the matrix $\Omega_{\alpha \beta}(t, x)$ since components of the latter are given by a change of variables (3.12). 
To restore the term $H$ in the Lagrange function (3.2), we need to solve the equation (3.7) with respect to $H$. We obtain for $H$ the following representation:

$$
H(t, x)=\int_{0}^{1} d s x^{\beta}\left[\Omega_{\beta \alpha}(t, s x) f^{\alpha}(t, s x)-\partial_{t} J_{\beta}(t, s x)\right]+c(t),
$$

where $c(t)$ is an arbitrary function of time, and $\Omega_{\beta \alpha}$ and $J_{\beta}$ are given by (3.12) and (3.17) respectively. All the arbitrariness in constructing $H$ is thus due to arbitrary symplectic matrix $\Omega_{\gamma \delta}^{(0)}$, arbitrary functions $\varphi(t, x)$ entering into $\Omega_{\beta \alpha}$ and $J_{\beta}$ and due $c(t)$.

We can see that there exist a family of actions (3.2) which lead to the same equations of motion (3.1). It is easy to see that actions with the same $\Omega_{\gamma \delta}^{(0)}$ but different functions $\varphi(t, x)$ and $c(t)$ differs by a total time derivative (we call such a difference trivial). A difference in Lagrange functions related to different choice of symplectic matrices $\Omega_{\alpha \beta}^{(0)}$ is not trivial. The corresponding Lagrangians are known as $s$-equivalent Lagrangians.

The first-order action (3.2) can be regarded as a Lagrangian action, or as a Hamiltonian action with a noncanonical Poisson bracket. An equivalent second-order Lagrangian formulation is always possible; however, it may include additional variables [20].

One ought to say that it is always possible to construct a Lagrangian action for non-Lagrangian second-order equations in an extended configuration space following a simple idea first proposed by Bateman [21]. Such a Lagrangian has the form of a sum of initial equations of motion being multiplied by the corresponding Lagrangian multipliers, new variables. The Euler-Lagrange equations for such an action contain besides the initial equations some new equations of motion for the Lagrange multipliers. In such an approach one has to think how to interpret the new variables already on the classical level. Additional difficulties (indefinite metric) can appear in course of the quantization.

As an example, let us consider a theory with equations of motion of the form ${ }^{1}$

$$
\dot{x}=A(t) x+j(t)
$$

and apply the above consideration to construct the action principle for such a theory.

The solution of the Cauchy problem for the equations (3.19) reads

$$
x(t)=\Gamma(t) x_{(0)}+\gamma(t)
$$

where the matrix $\Gamma(t)$ is the fundamental solution of (3.19), i.e.,

$$
\dot{\Gamma}=A \Gamma, \Gamma(0)=1
$$

and $\gamma(t)$ is a partial solution of (3.19). Then following (3.12), we construct the matrix $\Omega$,

$$
\Omega=\Lambda^{T} \Omega^{(0)} \Lambda, \Lambda=\Gamma^{-1} .
$$

and find the functions $J$ and $H$ according to (3.17) and (3.18),

$$
J=\frac{1}{2} x \Omega, H=\frac{1}{2} x B x-C x,
$$

\footnotetext{
${ }^{1}$ Here we use matrix notation, $x=\left(x^{\alpha}\right), A(t)=\left(A(t)_{\beta}^{\alpha}\right), j(t)=\left(j(t)^{\alpha}\right), \alpha, \beta=1, . ., 2 n$.
} 
where

$$
B=\frac{1}{2}\left(\Omega A-A^{T} \Omega\right), C=\Omega j .
$$

Thus, the action functional for the general quadratic theory is

$$
S[x]=\frac{1}{2} \int d t(x \Omega \dot{x}-x B x-2 C x) .
$$

One ought to say that an approach to constructing the action functional for the set linear inhomogeneous equations was proposed in [22].

\section{References}

[1] H. von Helmholtz, Journ. f. d. reine u. angew. Math., 100 (1887) 137.

[2] G. Darboux, Lesons sur la Theorie Generale des Surfaces (Gauther-Villars, Paris, 1894).

[3] J. Douglas, Trans. Am. Math. Soc.50, N 71 (1941) 71.

[4] W. Sarlet, J.Math.Phys.19(1978)1049.

[5] V.V. Dodonov, V.I. Man'ko and V.D. Skarzhinsky, Arbitrariness in the choice of action and quantization of the given classical eqations of motion, preprint of P.N. Lebedev Physical Institute (1978).

[6] W. Sarlet, J.Phys.A 15 (1982) 1503.

[7] A.P.Balachandran, T.R.Govindrajan and B.Vijayalakshimi, Phys.Rev. D18(1978)1950.

[8] S.Okubo, Phys.Rev. D22(1980)919.

[9] N.A. Lemos, Phys.Rev. D24(1981)1036.

[10] P. Havas, Actra. Phys. Aust. 38 (1973) 145.

[11] R. Santilli, Ann. Phys. 103 (1977) p.354.

[12] S. Hojman and L. Urrutia, J. Math. Phys. 22 (1981) 1896.

[13] S.Hojman and H.Handerston, J.Math.Phys. 22 (1981) 1414.

[14] M. Henneaux, Ann. Phys. 140 (1982) 45.

[15] M.Henneaux, L.Sheplley, J.Math.Phys 23 (1982) 2101.

[16] G. Morandi, C. Ferrario, G. Lo Vecchio, G. Marmo and C. Rubano, Phys. Rep. 188 (1990) 147.

[17] P.O. Kazinski, S.L. Lyakhovich and A.A. Sharapov, JHEP07(2005)076.

[18] D.M. Gitman, V.G. Kupriyanov, Canonical quantization non-Lagrangian systems theories and its application to a damped oscillator and a radiating point-like charge. [hep-th/0605025].

[19] V.G. Kupriyanov, Int.J.Theor.Phys.45 (2006) 1129.

[20] D.M. Gitman, S.L. Lyakhovich, M.D. Noskov and I.V. Tyutin, Izv VUZov 3 (1986).

[21] H. Bateman, Phys.Rev. 38 (1931) 815.

[22] V.G. Kupriyanov, S.L. Lyakhovich and A.A. Sharapov, J.Phys.A38 (2005) 8039. 